\title{
PREDICTIONS OF THE DYNAMIC RESPONSE OF PILED FOUNDATIONS IN A MULTI-LAYERED HALF-SPACE DUE TO INERTIAL AND RAILWAY INDUCED LOADINGS
}

\author{
Evangelos Ntotsios ${ }^{1}$, Waleed I. Hamad ${ }^{2}$, David J. Thompson ${ }^{1}$, Mohammed F.M. Hus- \\ sein $^{3}$, Hugh E.M. Hunt ${ }^{2}$, and James P. Talbot ${ }^{2}$ \\ ${ }^{1}$ Institute of Sound and Vibration Research, University of Southampton \\ Southampton, SO17 1BJ, U.K. \\ e-mail: e.ntotsios@ @oton.ac.uk; djt@isvr.soton.ac.uk \\ ${ }^{2}$ Department of Engineering, University of Cambridge \\ Trumpington Street, Cambridge CB2 1PZ, U.K. \\ \{wih22,hemh1,jpt1000\}@cam.ac.uk \\ ${ }^{3}$ Department of Civil and Architectural Engineering, College of Engineering, Qatar University \\ Doha, 2713, Qatar \\ mhussein@qu.edu.qa
}

Keywords: Pile Foundations, Pile-Soil-Pile Interaction, Ground Wave Propagation, Railway Induced Vibration.

\begin{abstract}
In this paper, the dynamic pile-soil-pile interaction (PSPI) in a multi-layered halfspace is investigated for the prediction of the response of piled foundations due to railway vibrations. Two methods of modelling piled foundations in a multi-layered half-space are presented. The first is an efficient semi-analytical model that calculates the Green's functions of the multi-layered half-space soil using the thin layer and the dynamic stiffness matrix methods. The second is a fully-coupled model that utilises the boundary element (BE) method to simulate the soil, where the Green's functions are calculated using the ElastoDynamics Toolbox (EDT). The paper aims to investigate the accuracy and the efficiency of the semi-analytical model by comparing the predictions of the two methods. A set of comparisons is performed, including the driving point response of a single pile and the interaction between two piles. The comparisons reveal that, at most frequencies, the semi-analytical model can predict the driving point response and the dynamic interaction with acceptable accuracy and computational efficiency. The model is then used for predicting the response of a pile-group due to the vibration field generated by a railway in varying distance from the piles. The vibration field generated by the railway is modelled as the superposition of the response due to harmonic loadings generated at the wheel-rail interface and the vibration response is examined at different points on the free surface away from the piles. The comparisons highlight the efficiency and accuracy of the semi-analytical model and illustrate its practical application.
\end{abstract}




\section{INTRODUCTION}

Ground-borne vibration generated by sources such as earthquakes, railways, roads and construction activities often causes disturbance to nearby buildings that may lead to significant social and economic impacts. Usually, structural foundations, such as piled foundations, act as a transmission path for ground-borne vibration into buildings. In some cases, however, piled foundations can serve as wave barriers to isolate vibration when arranged in appropriate configurations. As such, over the past four decades the dynamic pile-soil-pile interaction (PSPI) has received extensive research effort. Different techniques of modelling the dynamic PSPI have been formulated, most of them were recently reviewed by Kuo and Hunt [1].

The research interest in the dynamic PSPI was sparked by the pioneering work of Poulos $[2,3]$ on the static behaviour of pile groups. In his work, Poulos introduced the concept of interaction factors, which express the displacement of a pile group as a function of the motion of the loaded, adjacent pile. A number of researchers have later applied this concept to study the dynamic behaviour of pile groups, which was observed to be a strongly frequency dependent $[4,5]$. These findings, along with the increasing interest in soil-structure interaction, have led researchers to develop further analytical methods for modelling the dynamic behaviour of pile groups $[6,7]$. However, despite offering considerable reduction in computation time, the analytical models only provide approximate solutions for PSPI as the presence of neighbouring piles is ignored when calculating the interaction between two piles. The existence of neighbouring piles has two effects; the soil-stiffening effect, which dominates at low frequencies (wavelengths are greater than pile spacing), and the wave scattering effect, which dominates at high frequencies (wavelengths are less than the diameter of the piles) [8]. These shortcomings limit the application of such models in studying ground-borne vibration, and hence an efficient, yet rigorous analytical model is still required.

The boundary element (BE) method, on the other hand, is considered to be a reliable approach for modelling the dynamic PSPI. Unlike the finite element method, wave radiation is inherently accounted for, resulting in accurate solutions within the interior of the domain. For instance, Sen et al. [9] presented a BE model of a pile group, in which the piles are modelled as an elastic bar/beam and inserted into a homogeneous half-space with multiple cavities. Talbot and Hunt [8] avoided adding extra cavities in the soil by adopting instead the periodic structure theory to model an infinitely long row of piles embedded in a homogeneous halfspace. Maeso et al. [10] developed a pile group model by simulating the piles as continuous elastic solids and the soil as an isotropic homogeneous fluid-filled poroelastic material. More recently, Millán and Domínguez [11] devised a simplified BE model for pile groups in viscoelastic and poroelastic soils. All these previous BE models provide a good solution for PSPI and are capable of considering different types of motion of pile heads. BE models of piles, in general, can serve as a benchmark to scrutinise the effects of simplifying assumptions inherent in the analytical models. However, BE models are computationally inefficient and thus they may not be a suitable tool for design use by practicing engineers. This in turn shows the need for efficient models that account for essential aspects of the dynamic PSPI.

This paper presents two approaches of modelling piled foundations in a multi-layered halfspace. The first is an efficient semi-analytical model that simulates the soil as a horizontally layered semi-infinite system. The second is a fully-coupled model that utilises the BE method to model the soil. The pile is modelled, in both approaches, by an elastic bar for axial loading and an Euler-Bernoulli beam for transverse loading. The approaches adopted for modelling the ground are discussed in Section 2. In Section 3 the pile model and the coupling techniques are presented. The simulation parameters and results are presented in Section 4, and finally some findings and conclusions are highlighted in Section 5. 


\section{GROUND MODELLING}

To model the dynamic soil-structure interaction appropriately, it is essential to have an acceptable representation of the soil. Given the low strain amplitudes of ground-borne vibration, it is plausible to assume that the soil behaves in a linear-elastic manner. In the following sections, the two techniques adopted in the current work to model the ground, which both treat the soil as a linear-elastic medium, are described.

\subsection{Semi-analytical approach}

In the semi-analytical approach, the soil is modelled as a horizontally layered semi-infinite system and the response field is obtained by the use of integral transforms. For this formulation the source fields (displacements and tractions) are transformed to the wavenumber/frequency domain and the layered medium is modelled as an assembly of homogeneous layer and half-space elements. Closed-form solutions can be obtained to the Navier equations, governing wave propagation in a homogeneous layer or a homogeneous half-space, for each frequency and wavenumber. The response fields can be transformed back to the space/time domain by inverse Fourier transformation operations.

From the different available methods based on the wavenumber/frequency domain approach, a hybrid formulation of the Thin Layer Method (TLM) $[12,13]$ and the Direct Stiffness Matrix Method (DSMM) [12,14] is used for the calculation of the response field in the soil. In both these methods, which are similar to the finite element method, element stiffness matrices are used to express the relation between displacements and stresses at the soil element boundaries. The difference between the two methods is that the TLM is an approximate method using less computationally demanding polynomial functions whereas in the DSMM transcendental functions are used that are exact solutions for a given wavenumber and frequency. It should be noted that, due to its approximate nature, the TLM requires that the layer elements have a thickness that is small compared with the smallest relevant wavelength. This gives rise to systems of equations with more degrees of freedom than the DSSM. Nevertheless, the coupling between the pile and the soil introduces multiple source tractions in the soil along the length of the pile, so that each soil layer has to be divided to multiple sublayers. Consequently, the TLM is considered to be more suitable since it already offers high density of soil discretization with depth. Below the depth reached by the piles no more traction sources exist, so the soil layers and the homogenous half-space are modelled using DSMM. In addition this is necessary as the TLM is only applicable to media with a finite thickness.

Using the hybrid formulation and dropping for simplicity the dependence on the frequency $\omega$, the response $\mathbf{u}_{s}(r)$ of the soil (receiver) due to a load vector (source) $\mathbf{g}$ at a distance $r$ is given by

$$
\mathbf{u}_{s}(r)=\mathbf{H}_{h}(r) \mathbf{g}
$$

where $\mathbf{H}_{h}$ is the frequency response function (FRF) matrix relating displacements and tractions at the frequency of interest for an area or point load $\mathbf{g}$.

It should be noted that the soil with the cavity cannot be modelled using the semianalytical formulation. This means that effectively the volume of the piles is neglected, the interface between the pile and the soil being reduced to a line and the load delivered from the pile to the soil at each coupling node is distributed over an area. More details about the interaction between the pile and the soil is given in Section 3. 


\subsection{Boundary element approach}

In the second approach, the soil is modelled using the BE method, where threedimensional Green's functions for a multilayered half-space are obtained with the aid of the ElastoDynamics Toolbox (EDT) [12]. These fundamental solutions are calculated numerically based on the DSMM for wave propagation in layered media. A BE mesh is generated at the soil-structure interface to simulate a vertical cylindrical cavity. The mesh consists of a number of elements with nodal collocation, at which there are three values of displacements and three tractions. These variables are related at each collocation point $N_{S}$ by

$$
\mathbf{H u}=\mathbf{G p}
$$

where $\mathbf{H}$ and $\mathbf{G}$ are $3 N_{S} \times 3 N_{S}$ matrices describing the behaviour of the soil in terms of its density $(\rho)$, shear modulus $(\mu)$, Poisson's ratio $(v)$, damping ratio $(\eta)$, shear wave speed $\left(c_{S}\right)$, compressional wave speed $\left(c_{P}\right)$ and frequency of interest $(f)$. The $3 N_{S} \times 1$ vectors $\mathbf{u}$ and $\mathbf{p}$ are assembled from the complex displacement and traction amplitudes of each node as

$$
\begin{aligned}
\mathbf{u} & =\left[\begin{array}{lllllllllll}
u_{x}^{1} & u_{y}^{1} & u_{z}^{1} & u_{x}^{2} & u_{y}^{2} & u_{z}^{2} & \cdots & u_{x}^{N_{S}} & u_{y}^{N_{S}} & u_{z}^{N_{S}}
\end{array}\right]^{T} \\
\mathbf{p} & =\left[\begin{array}{lllllllllll}
p_{x}^{1} & p_{y}^{1} & p_{z}^{1} & p_{x}^{2} & p_{y}^{2} & p_{z}^{2} & \cdots & p_{x}^{N_{S}} & p_{y}^{N_{S}} & p_{z}^{N_{S}}
\end{array}\right]^{T}
\end{aligned}
$$

where $\mathbf{u}^{j}$ and $\mathbf{p}^{j}$ are the displacement and the traction vectors of node $j$.

Equation (2) is rearranged as,

$$
\begin{aligned}
\mathbf{u} & =\mathbf{H}^{-1} \mathbf{G p} \\
\mathbf{u} & =\mathbf{H}_{S} \mathbf{p}
\end{aligned}
$$

in which $\mathbf{H}_{s}$ is the FRF matrix relating displacements and tractions at the frequency of interest. To couple the soil's cavity to the pile, this FRF matrix is modified to relate displacements to forces instead of tractions by dividing it by the area of the corresponding elements in the $\mathrm{BE}$ mesh. Throughout the BE analysis, it is ensured that there are more than six elements for each wavelength to satisfy Domínguez recommendations [13] and achieve convergence.

\section{PILE MODELLING AND COUPLING}

The pile model is identical in both modelling approaches, and it is modelled as an elastic bar for axial loading and an Euler-Bernoulli beam for transverse loading. It is represented by its centroid axis, which has $N_{l}$ equally spaced nodes, Figure 1 . At each node, there are six degrees of freedom (DOF) representing displacements and rotations in the three directions. The pile is assumed to be unconstrained at its ends and any local deformation of the crosssection is neglected. Only the responses due to unit harmonic axial and transverse loads are given in this paper.

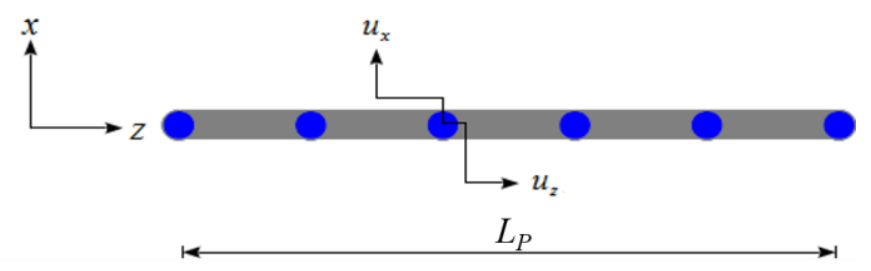

Figure 1: Pile centroid (drawn horizontally) where the circles represent the nodes at which the forces are applied and the responses are calculated. Only the $\mathrm{x}-\mathrm{z}$ plane is shown. 


\subsection{Pile response}

The response of the pile to a unit harmonic force with angular frequency $\omega$ applied in the vertical $z$ direction at node $j$ is calculated as

$$
\begin{aligned}
& u_{z}^{I}(z, \omega)=A^{I} \cos (\alpha z)+B^{I} \sin (\alpha z) \text { for } 0 \leq z \leq z^{j} \\
& u_{z}^{I I}(z, \omega)=A^{I I} \cos (\alpha z)+B^{I I} \sin (\alpha z) \text { for } z^{j} \leq z \leq L_{P}
\end{aligned}
$$

where $\alpha=\omega \sqrt{\rho_{P} / E_{P}}$ is the wavenumber, the superscripts $I$ and $I I$ indicate the sections above and below the excited node $j$ and the coefficients $A^{I}, A^{I I}, B^{I}$ and $B^{I I}$ are calculated from the boundary conditions. $L_{P}$ is the length, $\rho_{P}$ is the density and $E_{P}$ is Young's modulus.

The general response of the pile to a unit harmonic force with angular frequency applied in the transverse directions $(x, y)$ at node $j$ is given by

$$
\begin{aligned}
& u_{x, y}^{I}(z, \omega)=A^{I} \exp (\beta z)+B^{I} \exp (i \beta z)+C^{I} \exp (-\beta z)+D^{I} \exp (-i \beta z) \text { for } 0 \leq z \leq z^{j} \\
& u_{x, y}^{I I}(z, \omega)=A^{I I} \exp (\beta z)+B^{I I} \exp (i \beta z)+C^{I I} \exp (-\beta z)+D^{I I} \exp (-i \beta z) \text { for } z^{j} \leq z \leq L_{P}
\end{aligned}
$$

where $\beta=\left(\rho_{P} A_{P} \omega^{2} / E_{P} I_{P}\right)^{\frac{1}{4}}$ is the wavenumber and the coefficients $A^{I}-D^{I}$ and $A^{I I}-D^{I I}$ are calculated from the boundary conditions. $A_{P}$ is the cross-section area and $I_{P}$ is the second moment of inertia.

The FRF matrix of the pile's centroid $\mathbf{H}_{l}$ is assembled from the general responses in equations (5) and (6) for forces at each node in turn and the response of the pile due to a load vector $\mathbf{p}$ is given by

$$
\mathbf{u}_{l}=\mathbf{H}_{l} \mathbf{p}
$$

This FRF matrix is transformed, for the case of the BE approach, to give the FRF matrix $\mathbf{H}_{P}$ of the pile's nodes around the circumference as,

$$
\mathbf{H}_{P}=\mathbf{T}_{r}^{\prime} \mathbf{H}_{l} \mathbf{T}_{r}
$$

in which $\mathbf{T}_{r}$ is the transformation matrix, and the size of $\mathbf{H}_{P}$ is $3 N_{S} \times 3 N_{S}$.

\subsection{Coupling technique}

In the semi-analytical approach, two assumptions are adopted to perform the coupling between the soil and the pile. First, compatibility of displacements, i.e. the soil displacement $\mathbf{u}_{S}(r)$ in is equal to the pile displacement $\mathbf{u}_{l}$, and equilibrium of forces are assumed at the soil-pile interface. Second, the loads transmitted by the pile to each discretised ground layer are distributed either over a circular area of radius equal to that of the pile or over a circular annulus of width equal to one tenth of the pile radius. It should be noted that the calculation of the FRF due to an annulus loading is achieved by subtracting the FRF due to loading through the inner disc from the FRF due to loading through the outer disc.

Considering these assumptions, the coupling is achieved by combining equation (1) with

$$
\mathbf{u}_{l}=\mathbf{H}_{l}\left(\mathbf{f}_{a}-\mathbf{g}\right)
$$


that derives from equation (7) by substituting $\mathbf{p}=\mathbf{f}_{a}-\mathbf{g}$. In equation (9), $\mathbf{f}_{a}$ is the vector of applied forces at the pile centroid, and $\mathbf{g}$ is the vector of reaction forces between the pile and the soil, given by

$$
\left.\mathbf{g}=\left[\mathbf{H}_{h}(0)+\mathbf{H}_{l}\right)\right]^{-1} \mathbf{H}_{l} \mathbf{f}_{a} .
$$

Once $\mathbf{g}$ is known, the response at any point in the soil can be calculated using equation (1).

For the case of multiple piles, the soil response is given as a superposition of the displacement fields due to the tractions $\mathbf{g}_{i}$ of each individual pile $i$ as

$$
\mathbf{u}_{S}=\sum_{i=1}^{N} \mathbf{H}_{h}\left(r_{i}\right) \mathbf{g}_{i} .
$$

where $r_{i}$ is the distance between the receiver and $i$ th pile. For known applied forces $\mathbf{f}_{a i}$, the forces on the soil $\mathbf{g}_{i}$ are given by

$$
\mathbf{H}_{h}(0) \mathbf{g}_{i}+\mathbf{H}_{l} \mathbf{g}_{i}+\sum_{j=1}^{N} \mathbf{H}_{h}\left(r_{i j}\right) \mathbf{g}_{j}\left(1-\delta_{i j}\right)=\mathbf{H}_{l} \mathbf{f}_{a i} .
$$

where $r_{i j}$ is the distance between the and $i$ th and $j$ th pile, and $\delta_{i j}$ is the Kronecker delta.

In the BE approach, the coupling is again performed by applying at the interface compatibility of displacements and equilibrium of forces. This is obtained by combining the systems in equations (4) and (7) as,

$$
\begin{aligned}
& \mathbf{U}_{S}=\mathbf{H}_{S} \mathbf{F}_{\mathbf{S}} \\
& \mathbf{U}_{P}=\mathbf{H}_{P}\left(\mathbf{F}_{\alpha}-\mathbf{F}_{\mathbf{S}}\right) \\
& \mathbf{F}_{\mathbf{S}}=\left(\mathbf{H}_{S}+\mathbf{H}_{P}\right)^{-1} \mathbf{H}_{P} \mathbf{F}_{\alpha}
\end{aligned}
$$

where $\mathbf{F}_{\alpha}$ is the vector of forces applied to the pile circumference and $\mathbf{F}_{S}$ is the resulting vector of soil force, from which the response at any point in the soil can be calculated.

\section{PILE MODELLING AND COUPLING}

To compare the two modelling approaches, three cases are considered as shown in Figure 2. The pile has radius $R_{P}=0.5 \mathrm{~m}$, length $L_{P}=10 \mathrm{~m}$ and is made of concrete with Young's modulus $E_{P}=50 \mathrm{GPa}$, Poisson's ratio $v_{P}=0.3$, damping loss factor $\eta_{P}=0.01$, and density $\rho_{P}=2500 \mathrm{~kg} / \mathrm{m}^{3}$. The soil consists of four layers of increasing stiffness; the top three have thickness $d_{1}=2 \mathrm{~m}, d_{2}=4 \mathrm{~m}$ and $d_{3}=6 \mathrm{~m}$ while the fourth represents the half-space. All layers have density $\rho_{S}=2000 \mathrm{~kg} / \mathrm{m}^{3}$ and damping loss factor $\eta_{S}=0.06$ associated with both Lamé constants. The layers have shear wave velocities $c_{1}=[185,228,260,309] \mathrm{m} / \mathrm{s}$ and compressional wave velocities $c_{2}=[277,373,485,944] \mathrm{m} / \mathrm{s}$ respectively. 


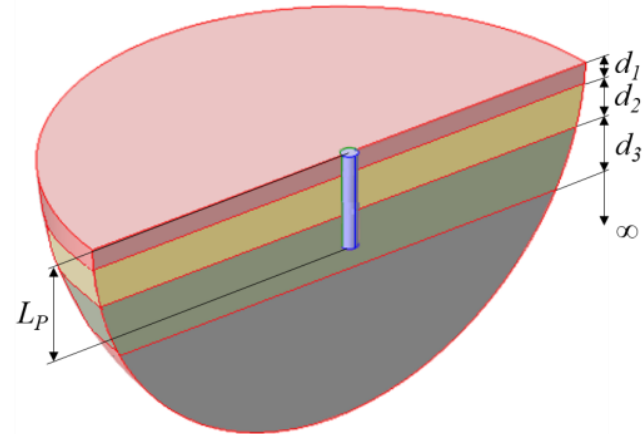

(a)

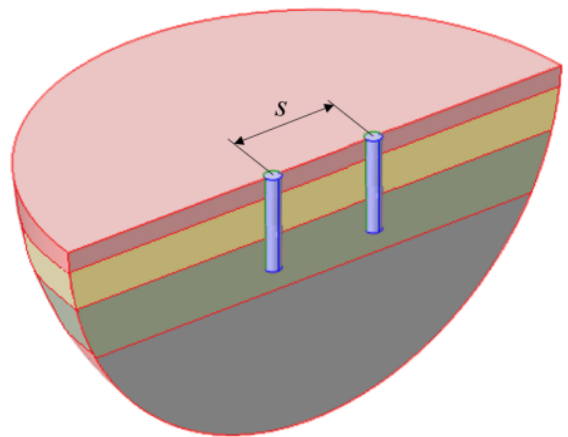

(b)

Figure 2: Pile in a multi-layered half-space: (a) single pile, (b) two piles with separation distance $s$.

\subsection{Free-surface displacement field}

The first set of results show the predictions of the BE model for the displacement field at the free surface for the cases in Figure 2 when one pile head is subject to a unit harmonic axial or transverse load at a frequency of $50 \mathrm{~Hz}$. In Figure 3(a), the vertical displacement field exhibits concentric circular wavefronts, which is expected when a single pile is subject to axial loading on its head. However, these circular wavefronts diffract when a second pile is inserted at $2 \mathrm{~m}$ in Figure 3(c) and at $4 \mathrm{~m}$ in Figure 3(e). This diffraction is more apparent in Figure 3(e) as the wavelength of the surface waves $(\sim 3.7 \mathrm{~m})$ is of the order of the separation distance. When the pile is subject to transverse load as in Figure 3(b), the horizontal displacement field does not show cylindrical wavefronts due to the nature of the source and the dynamic interaction between the soil and the pile. These wavefronts are not much influenced by the insertion of the pile at a distance $2 \mathrm{~m}$ as in Figure 3(d) where they slightly diffract. However, when a second pile is added at a distance $4 \mathrm{~m}$ the diffraction is more pronounced as shown in Figure $3(f)$.

\subsection{Comparison between the two models}

The comparisons between the models are given in terms of the driving point response of a single pile, and the interaction between two piles. The first is used to ensure that the semianalytical model is able to simulate the dynamic pile-soil interaction, whereas the other measures are utilised to scrutinise the dynamic PSPI. Since the BE model has been validated in [17] for a single pile, it is used to benchmark the semi-analytical model. For the semianalytical model both the case where the loads from the pile are transmitted to the ground layers distributed over a circular disc, and the case where they are distributed over an annulus are considered.

Figure 4, presents the driving point response of a single pile predicted by both models for axial and transverse unit harmonic loading. In general, very good agreement is seen between the semi-analytical model and the BE model. When considering the disc loading, the FRF due to axial loading (Figure 4(a)) of both models compares well up to $40 \mathrm{~Hz}$, above which an al most constant difference of less than $1 \mathrm{~dB}$ remains. In the FRF due to transverse load (Figure 4(b)), however, this difference begins at very low frequencies and continues to increase until it reaches about $2 \mathrm{~dB}$ at frequencies higher than $70 \mathrm{~Hz}$. For the annulus load the comparison of the FRF is much better for both axial and transverse loads. Nevertheless, when comparing the phase of the response between the two models, it is seen that the annulus load shows very good agreement with the BEM for the case of axial loading, but does not give such good agreement for the case of transverse loading. 

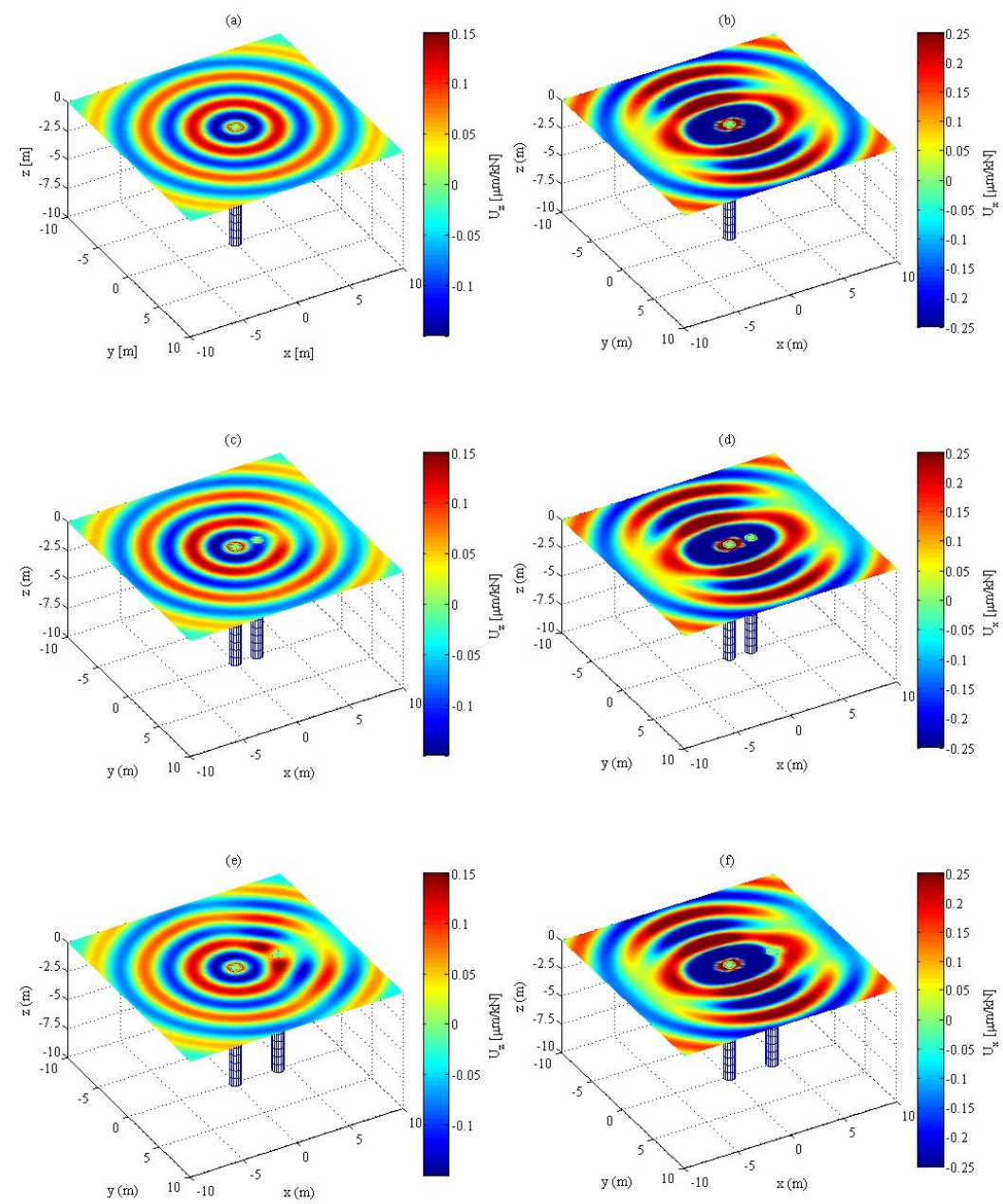

Figure 3: Pile Free-surface displacement field predicted by the BE model due to a unit harmonic load on the pile's head at a frequency $50 \mathrm{~Hz}$. (a), (c), (e) vertical response due to axial load and (b), (d), (f) horizontal response due transverse load for (a)-(b) single pile, (c)-(d) two piles with $2 \mathrm{~m}$ separation distance, and (e)-(f) two piles with $4 \mathrm{~m}$ separation distance.

In Figure 4, in general, it is seen that the FRF of the semi-analytical model is higher than that of the BE model, indicating that the semi-analytical model is softer even though it does not have a cavity as the BE model. This is believed to be due to the way of calculating the soil response in the semi-analytical model by applying circular or annulus load instead of a rigid disc load. The absence of the cavity in the semi-analytical model is believed to be another reason for these differences, which may lead to more inertia effects. This is apparent in the phase plots, where the differences reach about $5^{\circ}$. 

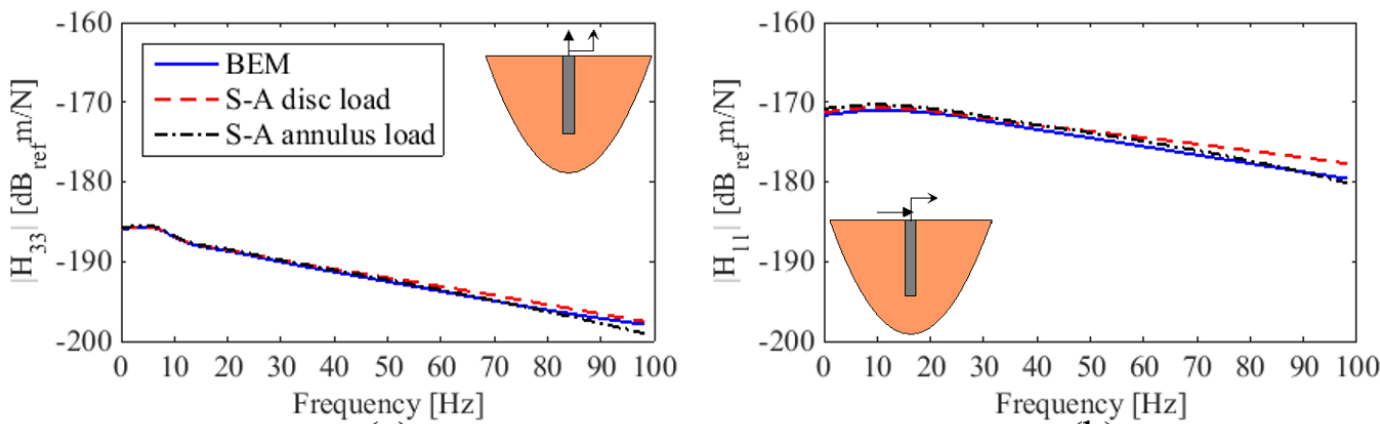

(a)

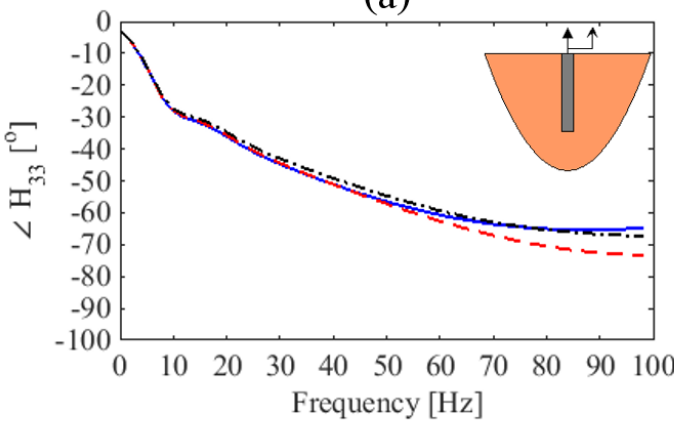

(c)

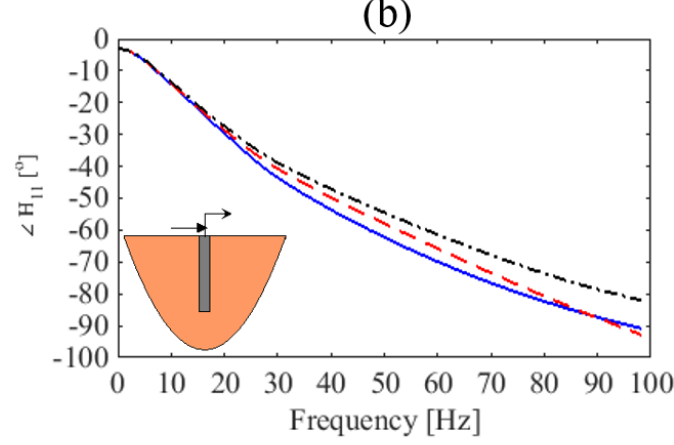

(d)

Figure 4: Driving point response of a single pile in a multilayered half-space due to (a)-(c) axial load and (b)-(d) transverse load predicted by the BE model (solid) and the semi-analytical model for disc loading (dashed) and annulus loading (dashed-dotted).
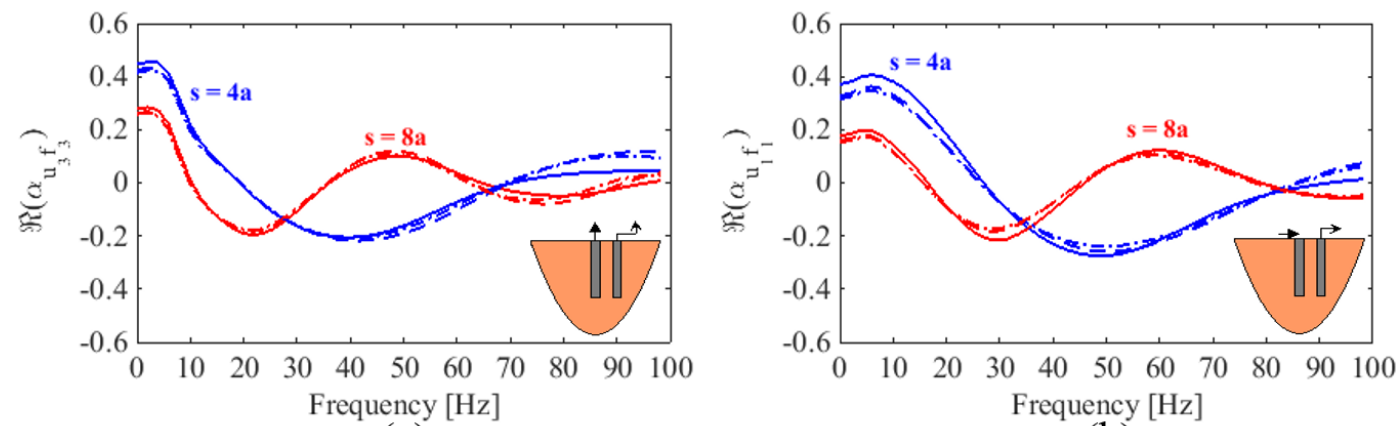

(a)

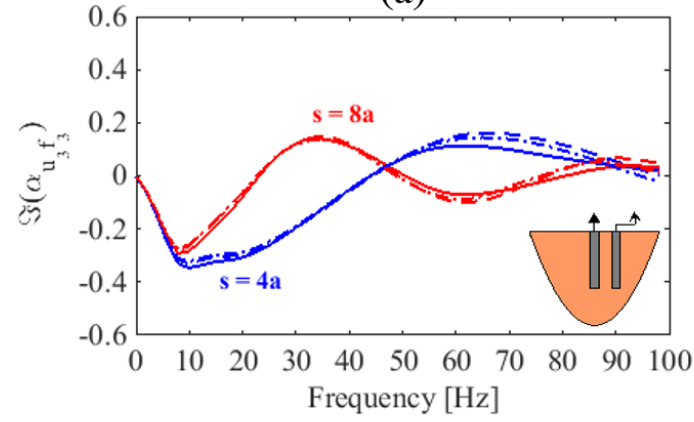

(c)

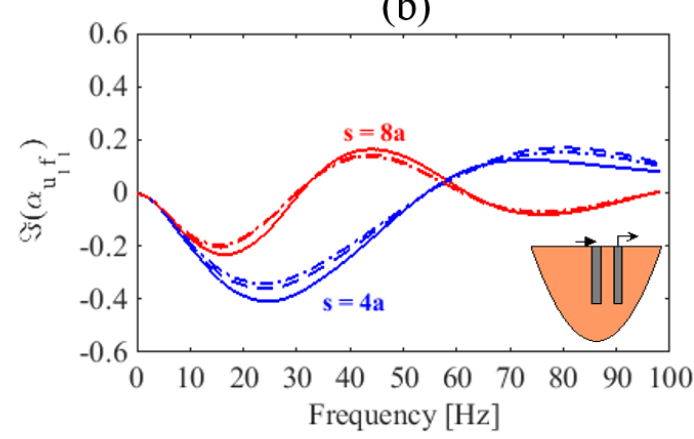

(d)

Figure 5: Dynamic interaction between two piles in a multi-layered half-space due to (a)-(c) axial load and (b)-(d) transverse load predicted by BE model (solid) and semi-analytical model for disc loading (dashed) and annulus loading (dashed-dotted). The piles' separation distances are $s=4 a$ and $s=8 a$, where $a$ is the pile radius. 
The interaction between two piles, which is calculated at the pile head, is presented by means of the dynamic interaction factor [2]

$$
\alpha_{u_{i} f_{i}}=\frac{u_{i_{b}}, f_{i_{a}}}{u_{i_{a}}, f_{i_{a}}}
$$

where $u_{i_{b}}, f_{i_{a}}$ is the displacement $i$ of pile $b$ due to load $i$ applied on pile $a$, and $u_{i_{a}}, f_{i_{a}}$ is the static displacement $i$ of pile $a$ due to load $i$ applied on pile $a$.

Figure 5, shows the dynamic interaction factors between two piles at separation distances $s$ of $2 \mathrm{~m}$ and $4 \mathrm{~m}$. Both real (top) and imaginary (bottom) parts of the dynamic interaction factors are given for axial and transverse loading. The predictions of the semi-analytical model are generally in good agreement with those of the BE model, in particular when the separation distance is $4 \mathrm{~m}$. For axial loading, discrepancies appear at frequencies higher than $50 \mathrm{~Hz}$, while discrepancies appear at very low frequencies for transverse loading, especially for the real part (Figure 5(b)). The reason for these discrepancies is believed to be twofold; one is due to the absence of the cavity in the analytical model and another is because of the procedure followed in calculating the soil response.

\subsection{Free-surface response in the existence of a pile group}

The semi-analytical model has shown it is capable of predicting the driving point response of a single pile and the dynamic PSPI with acceptable accuracy. In this section, the response of the free surface, generated by a vertical harmonic point source in the presence of a pile or a pile group is presented using the semi-analytical model. Since the vibration field generated by a railway is modelled as the superposition of the response due to harmonic loadings generated at the wheel-rail interface, the current investigation is the first important step for the developing of a fully coupled railway-soil-pile model using the semi-analytical approach. More effort is needed in order to couple a model of a railway track with the coupled soil-pile model that was developed in the current paper.

The results are presented in terms of the insertion gain (IG) between the displacements for the ground without any pile (free ground) and the displacements calculated with the existence of piles as

$$
I G=20 \log _{10} \frac{u_{i, \text { with }}}{u_{i, \text { free }}}
$$

where $u_{i, \text { with }}$ is the displacement with the pile and $u_{i, \text { free }}$ is the displacement of the free ground due to a point harmonic force.

The response is calculated at a distance of $8 \mathrm{~m}$ from the vertical point source and three cases are considered, as shown in Figure 6. In the first case, a single pile is included midway between the source and the receiver ( $4 \mathrm{~m}$ from the source). In the second case, the pile is at $6 \mathrm{~m}$ from the receiver and in the third case two piles, one at $4 \mathrm{~m}$ and one at $6 \mathrm{~m}$ from the source are considered. The soil and pile material properties used are the same as in the previous sections and only the disc loading is considered for the current application. 


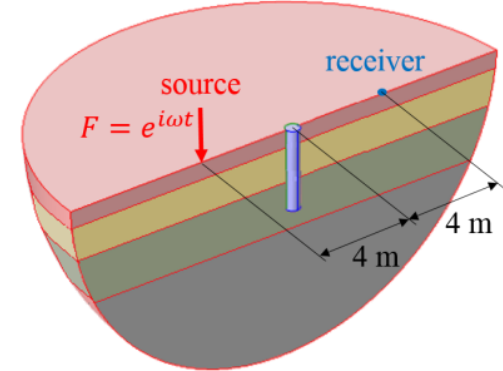

(a)

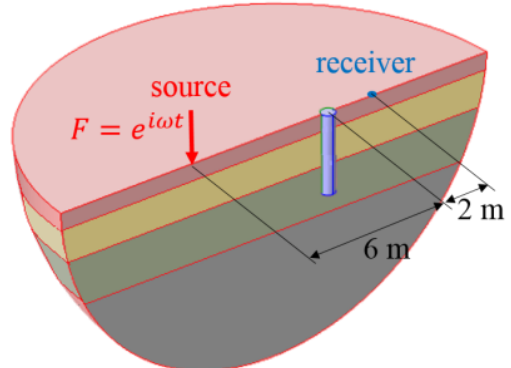

(b)

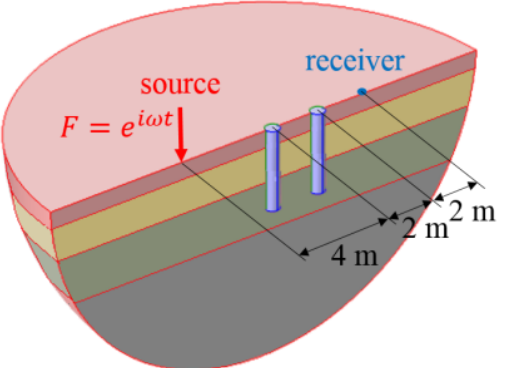

(c)

Figure 6: Pile(s) insertion cases between a point harmonic source and a receiver at $8 \mathrm{~m}$ : (a) single pile at $4 \mathrm{~m}$, (b) single pile at $6 \mathrm{~m}$, (b) two piles at $4 \mathrm{~m}$ and $6 \mathrm{~m}$.

Figure 7(a) and 7(b), compare the vertical and horizontal displacements respectively, for the three cases considered, with the displacement of the free ground. Figure 7(c) and (d) show the corresponding insertion gains IG. From these plots, it can be seen that the existence of the piles reduces significantly the displacements in both directions and this reduction increases at mid- frequencies where it can be up to $4 \mathrm{~dB}$ for the case of the pile inserted at $4 \mathrm{~m}$. The case where a single pile is inserted closer to the receiver ( $6 \mathrm{~m}$ from the source), shows 1 to $2 \mathrm{~dB}$ additional reduction compared with the case where the single pile is inserted at the mid-point $(4 \mathrm{~m})$. For the third case where two piles are inserted, the IG is, as expected, clearly better leading to an average reduction of about $4.5 \mathrm{~dB}$ in the whole frequency range for the vertical and about $7 \mathrm{~dB}$ in the range 10 to $60 \mathrm{~Hz}$ for the horizontal response.
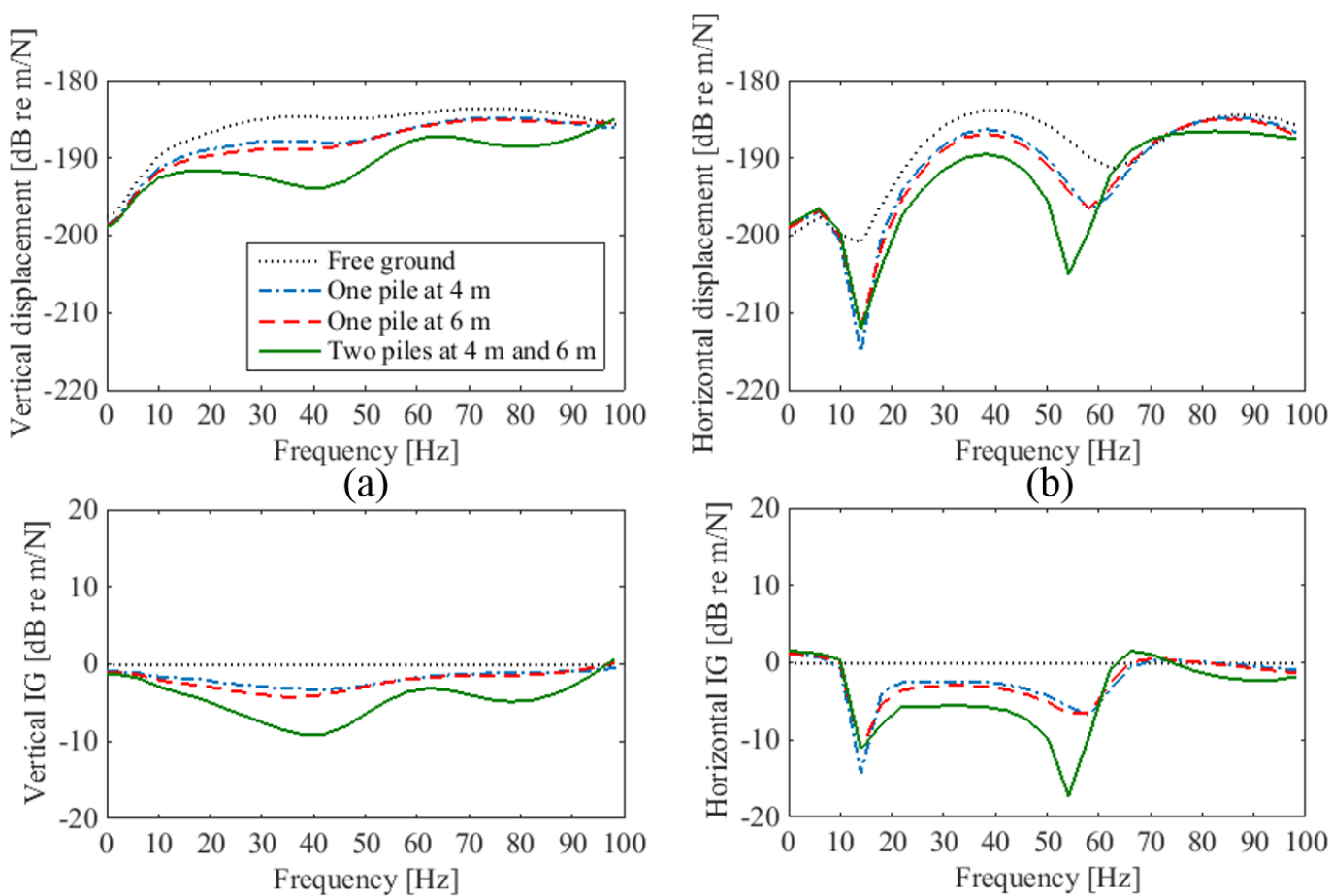

(b)

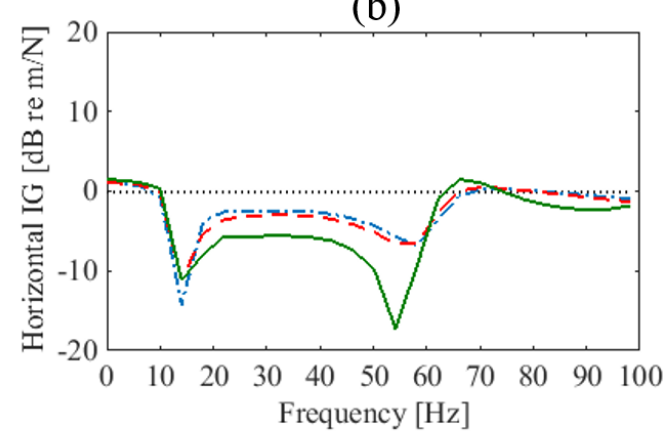

(c)

(d)

Figure 7: Vertical (a) and horizontal (b) FRF of the receiver at a distance $8 \mathrm{~m}$ from the source and IG of the vertical (c) and horizontal (d) displacement of the receiver for the free-ground (dotted), for the existence of a pile at $4 \mathrm{~m}$ (dashed-dotted), $6 \mathrm{~m}$ (dashed) and two piles at $4 \mathrm{~m}$ and $6 \mathrm{~m}$ (solid) from the source. 
Figure 7 showed that piled foundations can serve as wave barriers to isolate vibration in the far field which for the case of railway ground-borne vibrations can be used for vibration mitigation. It should be noted that for optimizing the performance of such piled wave barriers the piles should be arranged at appropriate distances and in appropriate configurations. Current modelling techniques can provide accurate and computationally efficient tools for the designing of such mitigation measures.

\section{CONCLUSIONS}

Investigating the dynamic interaction between piled foundations and soil is of great importance for predictions of ground-borne vibration. Such investigation entails comprehensive yet efficient models that consider essential aspects of the problem. This paper has presented an efficient semi-analytical model and a more versatile BE model to simulate the dynamic PSPI in multi-layered half-space. The models are compared together for predictions of the driving point response of a single pile subject to axial and transverse loading and the dynamic interaction between two piles. It has been demonstrated that the semi-analytical model captures the driving point response and the dynamic interaction between piles reasonably well, with some small differences at high frequencies. It is believed that the differences are due to (i) the absence of cavities in the semi-analytical model, which may provoke more inertia effects and (ii) the procedure followed in obtaining the soil response, which may result in a softer system. The semi-analytical model has then been used to predict the response of a pile-group due to the vibration field generated by a vertical load in varying distance from the piles. The example showed that the insertion of the piles in the wave propagation path can significantly reduce the level of vibration at the far field. Understanding and resolving the limitations of the semi-analytical model and coupling it with a detailed railway track model is the subject of ongoing work.

\section{ACKNOWLEDGEMENTS}

The authors would like to thank the EPSRC for funding this research, which is part of the MOTIV project (www.motivproject.co.uk) under the research grants EP/K006002/1 and EP/K006665/1. The authors also acknowledge the contribution of the Katholieke Universiteit Leuven in providing the EDT and also for some functions of BEMFUN used in the BE models.

\section{REFERENCES}

[1] K.A. Kuo, H.E.M. Hunt, Dynamic models of piled foundations. Applied Mechanics Reviews, 65(3), 031003-1-031003-9, 2013.

[2] H.G. Poulos, Analysis of the settlement of pile groups. Geotechnique, 18(4), 449-471, 1968.

[3] H.G. Poulos, Behaviour of laterally loaded piles: II-pile groups. ASCE Journal of Geotechnical Engineering Division, 97, 733-751, 1971.

[4] T. Nogami, Dynamic group effect of multiple piles under vertical vibration. Proceedings of the American Society of Civil Engineering Mechanics Division Speciality Conference, Austin, Texas, USA, 750-754, 1979. 
[5] M. Sheta, M. Novak, Vertical vibration of pile groups. ASCE Journal of Geotechnical Engineering Division, 108, 570-590, 1982.

[6] G. Gazetas, N. Makris, Dynamic pile-soil-pile interaction. Part 1: analysis of axial vibration. Earthquake Engineering and Structural Dynamics, 20, 115-132, 1991.

[7] A.M. Kaynia, E. Kausel, Dynamics of piles and pile groups in layered soil media. Soil Dynamics and Earthquake Engineering, 10(8), 386-401, 1991.

[8] J.P. Talbot, H.E.M. Hunt, A computationally efficient piled-foundation model for studying the effects of ground-borne vibration on buildings. Proc. the Institution of Mechanical Engineers, Part C: Journal of Mechanical Engineering Science, 217, 975-989, 2003.

[9] R. Sen, T.G. Davies, P.K. Banerjee, Dynamic analysis of piles and pile groups embedded in homogeneous soils. Earthquake Engineering and Structural Dynamics, 13, 53-65, 1985.

[10] O. Maeso, J.J. Anárez, F. García, Dynamic impedances of piles and pile groups in saturated soils. Computers and Structures, 83, 769-782, 2005.

[11] M.A. Millán, J. Domínguez, Simplified BEM/FEM model for dynamic analysis of structures on piles and pile groups in viscoelastic and poroelastic soils. Engineering Analysis with Boundary Elements, 33, 25-34, 2009.

[12] E. Kausel and J.M. Roësset, Stiffness matrices for layered soils. Bulletin of the Seismological Society of America, 71(6), 1743-1761, 1981.

[13] E. Kausel, R. Peek, Dynamic loads in the interior of a layered stratum: an explicit solution. Bulletin of the Seismological Society of America, 72(5), 1459-1481, 1982.

[14] E. Kausel, Fundamental solutions in elastodynamics: a compendium. Cambridge University Press, New York, 2006.

[15] M. Schevenels, S. Françcois, G. Degrande, EDT: An elastodynamics toolbox for MATLAB. Computers \& Geosciences, 35(80), 1752-1754, 2009.

[16] J. Domínguez, Boundary Elements in Dynamics. Computational Mechanics Publications and Elsevier Applied Science, Southampton, 1993.

[17] W.I. Hamad, H.E.M. Hunt, M.F.M. Hussein, J.P. Talbot, Tunnel-soil-pile interaction in the prediction of vibration from underground railways: validation of the sub-models. 9th International Conference on Structural Dynamics (EURODYN 2014), Porto, Portugal, 767-774, June 30-July 2,2014. 